McGill/93-06

hep-th/9305130

May 1993

\title{
Fractional Superspace Formulation of Generalized Mechanics
}

\author{
StÉPHANe DuranD* \\ Department of Physics \\ McGill University \\ 3600 University Street \\ Montréal, PQ, H3A 2T8, Canada
}

To appear in Mod. Phys. Lett. A8, No.24 (1993).

\begin{abstract}
Supersymmetric (pseudo-classical) mechanics has recently been generalized to fractional supersymmetric mechanics. In such a construction, the action is invariant under fractional supersymmetry transformations, which are the $F^{\text {th }}$ roots of time translations (with $F=1,2, \ldots$ ). Associated with these symmetries, there are conserved charges with fractional canonical dimension $1+1 / F$. Using paragrassmann variables satisfying $\theta^{F}=0$, we present a fractional-superspace formulation of this construction.
\end{abstract}

* E-mail address: durand@hep.physics.mcgill.ca 


\section{Introduction}

In field theory, supersymmetric (SUSY) transformations are "square roots" of space-time translations. As a special case, (quantum) mechanics can be considered as a field theory in one time and zero space dimensions, and in this context, SUSY transformations are then the square roots of time translations only. Since time translations are generated by the Hamiltonian $H$, and SUSY transformations by the supercharge $Q$, we are led to the algebra $H=Q^{2}$ which defines SUSY quantum mechanics.

We recently have presented a new generalization of SUSY quantum mechanics which we call fractional supersymmetric (FSUSY) quantum mechanics. ${ }^{[1,2]}$ In such a construction, the Hamiltonian is expressed as the $F^{\text {th }}$ power of a conserved fractional supercharge: $H=Q^{F}$, with $[H, Q]=0$ and $F=1,2, \ldots$. Motivated by this new algebra, we have pseudo-classically described FSUSY transformations as the $F^{\text {th }}$ roots of time translations, and provided an action invariant under such transformations. ${ }^{[1,3]}$ Here, we shall reformulate these results in fractional superspace, using paragrassmann variables of order $F$ satisfying $\theta^{F}=0$. Additionally, we construct the conserved Nöther fractional supercharges, which are of fractional canonical dimension $1+1 / F$, associated with these symmetry transformations. In the present work, we restrict ourselves to the free particle. The interacting case will be discussed elsewhere. ${ }^{[4]}$

\section{Fractional superspace formalism}

In this section, we introduce paragrassmann variables which interpolate between ordinary bosonic and fermionic variables, and which will be used to construct fractional-superspace coordinates. (In a quantum-mechanical context, these paragrassmann variables are instead interpreted as generalized creation and annihilation operators. ${ }^{[1,2]}$ ) Note that we will use the following definition:

$$
[A, B]_{\omega} \equiv A B-\omega B A
$$

We introduce a real paragrassmann variable $\theta$ of order $F$, and its (real) derivative $\partial \equiv \partial / \partial \theta$, which satisfy

$$
\begin{gathered}
\theta^{F}=0, \quad \partial^{F}=0, \quad F=1,2, \ldots \\
\left(\theta^{F-1} \neq 0, \partial^{F-1} \neq 0\right) .
\end{gathered}
$$


We take the generalized commutation relation between $\theta$ and $\partial$ to be

$$
[\partial, \theta]_{q}=\alpha(1-q)
$$

where $\alpha$ is an arbitrary real parameter, and $q \in \mathcal{C}$ a primitive $F^{\text {th }}$ root of unity:

$$
q^{F}=1 \quad\left(q^{n} \neq 1 \text { for } 0<n<F\right) .
$$

By a primitive root, we mean a root satifying the condition in parentheses; for instance, $q \neq \pm 1$ for $F=4$. We require the factor $\alpha(1-q)$ with a real (but arbitrary) $\alpha$ in the r.h.s of (3) so that (3) is consistent both under complex conjugation, and in the "null" case $F=1(q=1)$, i.e., $\theta=\partial=0$. Note that we recover the ordinary grassmann case for $F=2(q=-1): \theta^{2}=\partial^{2}=0$ and $\{\partial, \theta\}=2 \alpha$. Moreover, for some choices of $\alpha$ (for instance, $\alpha=F$ ), we also recover the bosonic case in the limit $F \rightarrow \infty(q=1):[\partial, \theta]=$ const. $^{[1,2]}$ However, in the following sections, we will not be concerned with this limit, so we let $\alpha$ remain unfixed. The definition (3) implies

$$
\partial \cdot \theta^{n}=\alpha\left(1-q^{n}\right) \theta^{n-1}+q^{n} \theta^{n} \partial .
$$

Setting $n=F$ in (5) demonstrates that the condition (4) is actually a consequence of (2) and (3).

For a given order $F$ which is prime, we can actually introduce $F-2$ other fractional derivatives. We thus have $F-1$ derivatives, which we write as $\partial_{i}$ with $i=1,2, \ldots, F-1$. With this notation, $\partial=\partial_{1}$. These derivatives satisfy

$$
\partial_{i}^{F}=0, \quad \partial_{i}^{F-1} \neq 0, \quad\left[\partial_{i}, \theta^{n}\right]_{q^{i n}}=\alpha\left(1-q^{i n}\right) \theta^{n-1}
$$

and have the properties

$$
\left[\partial_{i}, \partial_{F-i}\right]_{q^{-i}}=0 .
$$

Note that in the SUSY limit $(F=2 ; q=-1)$ we are left with only one derivative satisfying the usual relation: $[\partial, \partial]_{q^{-1}}=\{\partial, \partial\}=2 \partial^{2}=0$. For a non-prime $F$, the situation is more complicated. For instance, for $F=4$, setting $i=2$ in (7) implies $\left(\partial_{2}\right)^{2}=0$, which contradicts (6). In other words, $q^{i}$ is not a primitive root for $i=2$. Therefore, for a non-prime $F$, there are fewer than $F-1$ derivatives $\partial_{i}$, but at least two: $\partial_{\theta} \equiv \partial_{1}$ and $\delta_{\theta} \equiv \partial_{F-1}$. (We now put a $\theta$-subscript on the 
$\theta$-derivatives to clearly distinguish them from the time-derivative $\partial_{t} \equiv \partial / \partial t$ and from the variation $\delta$.) In the following, we will use only these two derivatives.

We now introduce the fractional covariant derivative

$$
D=\delta_{\theta}+i e \theta^{F-1} \partial_{t}, \quad D^{F}=i \partial_{t}
$$

and the generator of FSUSY transformations

$$
Q=\partial_{\theta}-i e \theta^{F-1} \partial_{t}, \quad Q^{F}=-i \partial_{t}
$$

where $e^{-1}=F \alpha^{F-1}$. These satisfy

$$
[D, Q]_{q}=0
$$

Note that in the SUSY case $(F=2)$, we have $\delta_{\theta}=\partial_{\theta}$ and $\{Q, D\}=0$. Note also that the null limit $\partial_{\theta}=0$ is needed for the consistency of (8) and (9) when $F=1$.

Finally, we define the integration over a (real) paragrassmann variable $\theta$ of order $F$ as

$$
\int d \theta \theta^{p}=\delta_{p, F-1}
$$

This direct generalization of integration over ordinary grassmann variables is invariant under translation $(\theta \rightarrow \theta+\epsilon)$, as in the grassmann case. Note however that unlike the grassmann case, the results of derivation and integration are different. Also note that there is no bosonic limit of such a definition. This is not a problem since we will not require the bosonic limit of a paragrassmann variable, but rather the bosonic limit of the fractional-superspace coordinate (which will correspond to the case $F=1$ ).

\section{Ordinary SUSY mechanics}

Let us first recall the ordinary SUSY case. We work in one dimension. The position of the particle is described by $x(t)$, whereas its internal space is described by a real fermionic variable $\psi(t)$ satisfying $\psi^{2}=0$. The SUSY transformations are given by

$$
\begin{aligned}
& \delta x=i \epsilon \psi \\
& \delta \psi=\epsilon \dot{x}
\end{aligned}
$$


where $\epsilon$ is a real fermionic infinitesimal parameter. Since $\epsilon \psi=-\psi \epsilon$, the r.h.s of Eqs. (12) are real. Note that $\delta^{2} x=i \epsilon_{1} \epsilon_{2} \dot{x}$ and $\delta^{2} \psi=i \epsilon_{1} \epsilon_{2} \dot{\psi}$, so SUSY transformations are indeed the square roots of time translations. An action invariant under (12) is

$$
S=\int d t \frac{1}{2}\left(\dot{x}^{2}+i \dot{\psi} \psi\right)
$$

More precisely, the Lagrangian of (13) varies by the total time derivative $\partial_{t}\left(\frac{i}{2} \dot{x} \psi\right)$. Note that since $\psi \dot{\psi}=-\dot{\psi} \psi$, this action is real.

To give a superspace formulation of this construction, we need a real grassmann coordinate $\theta$ and its derivative $\partial_{\theta}$ which satisfy

$$
\theta^{2}=\partial_{\theta}^{2}=0, \quad\left\{\partial_{\theta}, \theta\right\}=1
$$

these are the relations $(2-4)$ for $F=2$ (and $\alpha=1 / 2)$. We also recall the rule of integration over ordinary grassmann variables:

$$
\int d \theta(a+\theta b)=b
$$

Then, we combine the variables $x$ and $\psi$ into a superspace coordinate $Z(t, \theta)$ :

$$
Z(t, \theta)=x(t)+i \theta \psi(t)
$$

We say that $x$ and $\psi$ respectively belong to the sector- 0 and sector- 1 of the theory ( $\theta$ is also in sector- 1 and sectors are defined modulo 2$)$. Note that $Z$ is real since $\psi \theta=-\theta \psi$. In this formalism, the SUSY transformations are generated by

$$
Q=\partial_{\theta}-i \theta \partial_{t}, \quad Q^{2}=-i \partial_{t}
$$

Acting on $Z(t, \theta)$, we have

$$
\delta Z=\epsilon Q Z
$$

which gives in components the transformations (12). We also need the covariant derivative

$$
D=\partial_{\theta}+i \theta \partial_{t}, \quad D^{2}=i \partial_{t}
$$

which anticommutes with $Q$ :

$$
\{D, Q\}=0 \text {. }
$$


The relation (20) implies that if $A$ transforms as $\delta A=\epsilon Q A$ (i.e., if $A$ is a superfield), then $D A$ transforms in the same way, $\delta(D A)=D(\delta A)=D(\epsilon Q A)=$ $\epsilon Q(D A)$, since $\epsilon D=-D \epsilon$ (i.e., since $\epsilon$ anticommutes with $\theta$ and $\left.\partial_{\theta}\right)$. Thus, the covariant derivative of a superfield is again a superfield. Moreover, the product of two superfields is also a superfield since the Leibniz rule holds:

$$
\epsilon Q(A B)=\epsilon Q(A) B+A \epsilon Q(B) .
$$

Indeed, we have $\delta(A B)=(\delta A) B+A(\delta B)=(\epsilon Q A) B+A(\epsilon Q B)=\epsilon Q(A B)$. Therefore, it is easy to construct an invariant action. For instance, the action

$$
S=-\int d t d \theta \frac{1}{2}\left(D^{2} Z D Z\right)=-i \int d t d \theta \frac{1}{2}(\dot{Z} D Z)
$$

is invariant under (18) since $\delta S=-\frac{1}{2} \int d t d \theta \epsilon Q\left(D^{2} Z D Z\right)$, which is automatically zero (as we will see in Sect. 5 for the general case). Using the rule (15) to integrate over $\theta$, we find the action (13).

\section{FSUSY mechanics of order 3}

Let us now turn to the next order: we now want to construct an action invariant under cube roots of time translations. We first introduce the fractionalsuperspace coordinate $Z(t, \theta)$ of order $3:^{\dagger}$

$$
Z(t, \theta)=x(t)+q^{1 / 2} \theta \psi(t)+q^{2} \theta^{2} \phi(t)
$$

where $\theta$ is a real paragrassmann variable satisfying $\theta^{3}=0$, and where $q$ is a primitive cube root of unity, i.e., one which satisfies $q^{3}=1$ with $q \neq 1$. The factors $q^{1 / 2}$ and $q^{2}$ in (23) are needed in order for $Z$ to be real (see below). We thus have a theory with three sectors, and sectors are defined modulo 3 . The bosonic variable $x(t)$ remains a sector-0 quantity, but now we have two types of real internal-space variables, $\phi(t)$ and $\psi(t)$, which respectively belong to sector-1 and sector- 2 since we take $\theta$ to be in sector- 1 (and since $Z(t, \theta)$ must be a sector-0 quantity). We take the following commutation relations between the new variables:

$$
\theta \phi=q \phi \theta, \quad \theta \psi=q^{2} \psi \theta
$$

and

$\dagger$ Throughout this paper, we will use the following convention: for $q=e^{i \theta}$ with $0 \leq \theta<2 \pi$, the square root is defined as $q^{1 / 2}=e^{i \theta / 2}$. 


$$
\psi \dot{\phi}=q \dot{\phi} \psi, \quad \phi \dot{\psi}=q^{2} \dot{\psi} \phi
$$

We now can see that $Z^{*}=Z$. Since we have chosen to work with a real paragrassmann variable $\theta$, the series in (23) contains only 3 terms and no auxiliary field. [Alternatively, we could have chosen to work with a complex $\theta$, we then would have had a fractional-superspace coordinate $Z\left(t, \theta, \theta^{*}\right)$ containing 9 terms, among which some would have been auxiliary fields. But here we want to present the construction in the simpler formulation of (23).]

The FSUSY transformations of order 3 are the cube roots of a time translation. They are generated by the FSUSY generator

$$
Q=\partial_{\theta}-i e \theta^{2} \partial_{t}, \quad Q^{3}=-i \partial_{t}
$$

where $e^{-1}=3 \alpha^{2}$, and where $\theta$ and $\partial_{\theta}$ satisfy (2-4) for $F=3$. Acting on $Z(t, \theta)$, we have

$$
\delta Z=\left(i q^{-1 / 2}\right) \epsilon Q Z
$$

which gives in components

$$
\begin{aligned}
& \delta x=i \epsilon \alpha(1-q) \psi \\
& \delta \psi=i \epsilon \alpha\left(1-q^{2}\right) \phi \\
& \delta \phi=\epsilon e \dot{x}
\end{aligned}
$$

where $\epsilon$ is a real sector- 1 infinitesimal parameter, which must satisfies ${ }^{\dagger}$

$$
\epsilon \theta=q^{-1} \theta \epsilon, \quad \epsilon \partial_{\theta}=q \partial_{\theta} \epsilon, \quad \epsilon \delta_{\theta}=q \delta_{\theta} \epsilon
$$

In order for the r.h.s of (27) to be real, $\epsilon$ must satisfy the following commutation relations with the different fields:

$$
\epsilon x=x \epsilon, \quad \epsilon \phi=q \phi \epsilon, \quad \epsilon \psi=q^{2} \psi \epsilon .
$$

We easily see that $\delta^{3}=-\epsilon_{1} \epsilon_{2} \epsilon_{3} \partial_{t}$ and thus Eqs. (27) indeed represent cube roots of time translations. An action invariant under (27) is

$$
S=\int d t \frac{1}{2}\left[\dot{x}^{2}+i \beta(1-q) \dot{\phi} \psi+i \beta\left(1-q^{2}\right) \dot{\psi} \phi\right]
$$

\footnotetext{
$\dagger$ To pass from (26) to (27), we need to use either $(24 a)$ or $(28)$.
} 
with $\beta=3 \alpha^{3}$. This Lagrangian is real according to $(24 b)$, and varies by a total derivative under the transformations (27) [see below]. In Sect. 6, we will construct the associated conserved fractional charge.

Let us now write the action (30) in a fractional-superspace formulation, in order to make its FSUSY invariance manifest. First, we define integration over a paragrassmann variable of order 3 as

$$
\int d \theta\left(a+\theta b+\theta^{2} c\right)=c
$$

which is the particular case $F=3$ of (11). Then, we introduce the fractional covariant derivative of order 3

$$
D=\delta_{\theta}+i e \theta^{2} \partial_{t}, \quad D^{3}=i \partial_{t}
$$

which satisfies the commutation relation (10) with $Q$. For $D Z$ to transform in the same way as $Z$ in (26), we must have $[\epsilon Q, D]=0$, i.e. $\epsilon D=q D \epsilon$, which follows from (28). Moreover, according to (24a), (28) and (29), we can show that the Leibniz rule (21) still holds. ${ }^{\dagger}$ Hence, the fractional covariant derivative of a fractional superfield is also a fractional superfield, and the product of two fractional superfields is again a fractional superfield. For instance, the action

$$
S=-\int d t d \theta \frac{1}{2 e}\left(D^{3} Z D Z\right)=-i \int d t d \theta \frac{1}{2 e}(\dot{Z} D Z)
$$

is invariant under (26) since the variation of the Lagrangian can be written as a total $Q$-derivative, which implies $\delta S=0$ (as we will see in Sect. 5). Integrating out the paragrassmann variable $\theta$ via the rule (31), we obtain the action (30).

\section{FSUSY mechanics of arbitrary order}

Let us now turn to the general case, i.e., the $F^{\text {th }}$ roots of time translations with $F=1,2, \ldots$. We introduce the fractional-superspace coordinate $Z(t, \theta)$ of order $F$ :

$$
Z(t, \theta)=\sum_{i=0}^{F-1} c_{i} \theta^{i} \psi_{(i)}=x(t)+\sum_{i=1}^{F-1} c_{i} \theta^{i} \psi_{(i)}, \quad c_{i}=q^{i^{2} / 2}
$$

$\dagger$ The Leibniz rule works differently for $D$ and $Q$, as we will see in Sect. 6 . 
where $\theta$ is a real paragrassmann variable satisfying $\theta^{F}=0$, where $\psi_{(0)} \equiv x(t)$, and where $q$ is a primitive $F^{\text {th }}$ root of unity [i.e., satisfies (4)]. Since we take $\theta$ to be in sector-1, the real fields $\psi_{(i)}=\psi_{(i)}(t)$ belong to the sector- $(F-i)$. Sectors are defined modulo $F$. Note that the case $F=1$ corresponds to an ordinary bosonic variable. We introduce the following commutation relations:

$$
\begin{aligned}
\theta \psi_{(i)} & =q^{-i} \psi_{(i)} \theta \\
\psi_{(i)} \psi_{(F-i)} & =q^{i} \psi_{(F-i)} \psi_{(i)} .
\end{aligned}
$$

The relation (35a) implies that $Z^{*}=Z$. Consistent with (35b), we also introduce the commutation relation

$$
\psi_{(i)} \dot{\psi}_{(F-i)}=q^{i} \dot{\psi}_{(F-i)} \psi_{(i)}
$$

For $F=2$, the relations (35) and (36) reduce to the proper SUSY results $\theta \psi=$ $-\psi \theta, \psi^{2}=0$ and $\psi \dot{\psi}=-\dot{\psi} \psi$ [where $\psi \equiv \psi_{(1)}$ ]. With the identification $\psi_{(F)} \equiv x$, the relation (36) also implies $\dot{x} x=x \dot{x}$.

The FSUSY transformations of order $F$, i.e. the $F^{\text {th }}$ roots of time translations, are generated by the FSUSY generator $Q$ given in (9). It belongs to the sector$(F-1)$ since $\theta$ and $\partial_{\theta}$ belong respectively to the sectors 1 and $F-1$. The variation (26) now gives in components $(i=1,2, \ldots, F-1)$ :

$$
\begin{aligned}
& \delta \psi_{(i-1)}=i \epsilon \alpha\left(1-q^{i}\right) \psi_{(i)} \\
& \delta \psi_{(F-1)}=\epsilon e \dot{x}
\end{aligned}
$$

where the real infinitesimal parameter $\epsilon$ belongs as before to the sector-1, and satisfies (28). We now have $\delta^{F} \psi_{(i)}=i^{F-1} \epsilon_{1} \ldots \epsilon_{F} \dot{\psi}_{(i)}\left[\right.$ since $\left.\prod_{i=1}^{F-1}\left(1-q^{i}\right)=F\right]$, and thus the transformations $(37)$ are indeed $F^{\text {th }}$ roots of time translations. To ensure that the r.h.s of the transformations (37) are real (and that the action given below is invariant), we must take the following commutation relations between $\epsilon$ and $\psi_{(i)}$ :

$$
\epsilon \psi_{(i)}=q^{-i} \psi_{(i)} \epsilon
$$

For later convenience, we may combine the equations $(37)$ as $(i=0,1, \ldots, F-1)$ :

$$
\delta \psi_{(i)}=i \epsilon \alpha\left(1-q^{i+1}\right) \psi_{(i+1)}+\epsilon e \dot{x} \delta_{i, F-1} .
$$


Our previous results (12) and (27) are recovered for $F=2$ and $F=3$, with the notation $\psi_{(1)} \equiv \psi$ and $\psi_{(2)} \equiv \phi$. For $F=1$, we simply have $\delta x=\epsilon \dot{x}$. Note that in (26), $i q^{-1 / 2}=1$ for $F=2$. An action invariant under (39) is:

$$
S=\int d t \frac{1}{2}\left[\dot{x}^{2}+i \beta \sum_{i=0}^{F-1}\left(1-q^{-i}\right) \dot{\psi}_{(i)} \psi_{(F-i)}\right]
$$

with $\beta=\alpha e^{-1}=F \alpha^{F}$. More precisely, the Lagrangian varies by the total derivative

$$
\delta L=\frac{d}{d t} \frac{\epsilon}{2}\left[i \alpha(1-q) \dot{x} \psi_{(1)}+\dot{x}^{2} \delta_{F, 1}\right] \equiv \frac{d}{d t} X .
$$

Alternatively, using (36) and the symmetry of the action under the substitution $i \rightarrow F-i$, we may rewrite $(40 a)$ as

$$
S=\int d t \frac{1}{2}\left[\dot{x}^{2}-i \beta \sum_{i=0}^{F-1}\left(1-q^{-i}\right) \psi_{(i)} \dot{\psi}_{(F-i)}\right] .
$$

Note that the action is real. The first term of the sum in (40) always vanishes, but is included because it allows us to take the $F=1$ limit, which corresponds to the spinless (free) particle:

$$
S=\int d t \frac{1}{2} \dot{x}^{2}
$$

The cases $F=2($ with $\alpha=1 / 2)$ and $F=3$ reduce to those given previously in (13) and (30).

The fractional-superspace formulation of the action is:

$$
S=-\int d t d \theta \frac{1}{2 e}\left(D^{F} Z D Z\right)=-i \int d t d \theta \frac{1}{2 e}(\dot{Z} D Z)
$$

where $D$ is given in (8). In such a formulation, a general action is invariant under a transformation $Z \rightarrow Z+\delta Z$ if the variation of the Lagrangian can be written as a total derivative,

$$
\delta L=\partial_{t} \Lambda_{1}+\partial_{\theta} \Lambda_{2}+\delta_{\theta} \Lambda_{3}
$$

since the time-integrations of $\partial_{t} \Lambda_{1}$ and the $\theta$-integrations of $\partial_{\theta} \Lambda_{2}$ and $\delta_{\theta} \Lambda_{3}$ all vanish (there is no $\theta^{F-1}$ term in $\partial_{\theta} \Lambda_{2}$ and $\delta_{\theta} \Lambda_{3}$ which would have survived the $\theta$-integration). In particular, a variation of the Lagrangian which is a total $Q$ derivative or $D$-derivative,

$$
\delta L=Q(\Lambda)+D\left(\Lambda^{\prime}\right)
$$


can be written as in (44). Therefore, the action (43) is automatically invariant under the variation $(26)$ since $\delta(\dot{Z} D Z)=\epsilon Q(\dot{Z} D Z)$; to see this last point, recall the discussion between formulas (32) and (33), replacing (24a) and (29) by (35a) and (38). Using the rule (11) to integrate over $\theta$ in (43), we obtain the action (40). In order to recover the action of a spinless particle (42) directly from (43) for $F=1$, we need to define the integration over a paragrassmann variable of order 1 as $\int d \theta=1$, i.e., as the $F=1$ case of the formula (11).

\section{Fractional supercharges and Euler-Lagrange equations}

We first introduce the generalized momenta conjugate to $\psi_{(i)}$ :

$$
\begin{aligned}
& \pi_{(0)} \equiv \frac{\partial L}{\partial \dot{x}}=\dot{x} \\
& \pi_{(i)} \equiv 2 \frac{\partial L}{\partial \dot{\psi}_{(i)}}=i \beta\left(1-q^{-i}\right) \psi_{(F-i)}, \quad i=1, \ldots, F-1
\end{aligned}
$$

(the factor 2 will be discussed below). They are the components of the fractionalsuperspace momentum conjugate to $Z(t, \theta)$

$$
\Pi(t, \theta) \equiv 2 \frac{\partial L}{\partial \dot{Z}}=-\frac{i}{e} D Z
$$

which is decomposed as

$$
\Pi(t, \theta)=\sum_{i=0}^{F-1} a_{i} \theta^{i} \pi_{(F-1-i)}, \quad a_{i}=q^{\left(i^{2}-1\right) / 2} .
$$

Note that $\Pi^{*}=\Pi$. In order to recover the proper result for $F=1$, i.e. $p=\dot{x}$, we

must consider $\dot{Z}$ and $D Z$ as independent variables when calculating (47) even for $F=1$. The action is then simply

$$
S=\int d t d \theta \frac{1}{2}(\dot{Z} \Pi)
$$

We also have

$$
\int d \theta[\Pi, Z]=0 \quad \text { and } \quad \int d \theta[\Pi, \dot{Z}]=0
$$

which follow from (35). If we wish to add

$$
[\dot{Z}, Z]=0
$$


we must require

$$
\psi_{(i)} \dot{\psi}_{(j)}=\dot{\psi}_{(j)} \psi_{(i)} \quad \text { for } \quad j \neq F-i
$$

We now wish to construct the conserved Nöther charges associated with the symmetry transformations (39). It is important to notice that for a general Lagrangian of the form $L=\sum_{i=0}^{F-1} \alpha_{i} A_{(i)} B_{(F-i)}$ with $A_{(i)} B_{(F-i)}=q^{ \pm i} B_{(F-i)} A_{(i)}$ (we concentrate here on the internal-space part), we have

$$
\delta L \equiv \sum_{i=0}^{F-1} \alpha_{i}\left[\delta A_{(i)} B_{(F-i)}+A_{(i)} \delta B_{(F-i)}\right]=\sum_{i=0}^{F-1}\left[\delta A_{(i)} \frac{\partial L}{\partial A_{(i)}}+\delta B_{(i)} \frac{\partial L}{\partial B_{(i)}}\right]
$$

Then, it is easy to show that the generalized Euler-Lagrange equations which follow from a least-action principle are

$$
\frac{\partial L}{\psi_{(i)}}-\frac{d}{d t}\left(\frac{\partial L}{\dot{\psi}_{(i)}}\right)=0
$$

Therefore, the quantity

$$
C=\sum_{i=0}^{F-1} \delta \psi_{(i)} \frac{\partial L}{\partial \dot{\psi}_{(i)}}-X, \quad \frac{d C}{d t}=0
$$

is a constant of motion when the Lagrangian varies under a transformation $\delta \psi_{(i)}$ by the total derivative $\delta L=d X / d t$. The Hamiltonian is simply the particular case $\delta \psi_{(i)}=\dot{\psi}_{(i)}$ :

$$
H=\sum_{i=0}^{F-1} \dot{\psi}_{(i)} \frac{\partial L}{\partial \dot{\psi}_{(i)}}-L=\frac{1}{2} \dot{x}^{2}
$$

For $\delta \psi_{(i)}$ given by (39) and corresponding $X$ given in (41), we find the following conserved fractional supercharge: ${ }^{\dagger}$

$$
\mathbf{Q}=\frac{1}{2} \sum_{i=0}^{F-1} \pi_{(F-1-i)} \pi_{(i)}
$$

$\dagger$ If we had defined $\pi_{(i)}$ in (46b) without the factor 2 , the boundaries of the summation in (57) would have been $\sum_{i=1}^{F-1}$, whereupon the case $F=1$ would not have been included. Moreover, the definitions (46) are those which follow naturally from the fractional-superspace formulation (47). 
The three first orders are explicitly:

$$
\begin{array}{ll}
F=1: & \mathbf{Q}=\frac{1}{2} \dot{x}^{2} \\
F=2: & \mathbf{Q}=a \dot{x} \psi \\
F=3: & \mathbf{Q}=b \dot{x} \psi+c \phi^{2}
\end{array}
$$

where

$$
a=2 i \beta, \quad b=i \beta(1-q), \quad c=\frac{3}{2} \beta^{2} q^{2} .
$$

Note that $(\epsilon \mathbf{Q})^{*}=\epsilon \mathbf{Q}$, i.e., $\mathbf{Q}^{*}=q^{-1} \mathbf{Q}$, using $(52)$; however, $q^{-1 / 2} \mathbf{Q}$ is a real charge. For $F=1, \mathbf{Q}$ is simply the Hamiltonian, and for $F=2$ we recover the usual SUSY charge. The canonical dimension of $\mathbf{Q}$ is $\Delta[\mathbf{Q}]=1+1 / F$ (we have $\Delta\left[\partial_{t}\right]=1, \Delta[\theta]=-1 / F$ and $\left.\Delta\left[\psi_{(i)}\right]=i / F\right)$. It would be natural to guess that other FSUSY transformations are generated by different powers of $Q$, i.e., $Q^{k}$ with $k=1, \ldots, F$. However, if one tries to apply the Leibniz rule (21) with $\epsilon^{\prime} Q^{k}$, one finds that it holds only for $k=1$ and $k=F$, i.e., for $Q$ and $\partial_{t}$. Therefore, there are conserved charges with dimension $\Delta=1+k / F$ only for $k=1$ and $k=F$. Note also that the charge $\mathbf{Q}$ can be seen as a "one time and zero space" version of a conserved fractional current. On the other hand, the generator $Q$ of FSUSY transformations is one element of an infinite algebra which we call fractional superVirasoro algebra. ${ }^{[5]}$ In this context, $Q$ has fractional "conformal spin" $s=1+1 / F$.

Finally, let us write the fractional-superspace formulation of the Euler-Lagrange equation of motions. We first emphasize that in order for the operator $\hat{\epsilon} D$ to obey the Leibniz rule, as $\epsilon Q$ in (21), the parameter $\hat{\epsilon}$ must be different from $\epsilon$ [which satisfies (28)]. Rather, we have

$$
\hat{\epsilon} D(A B)=\hat{\epsilon} D(A) B+A \hat{\epsilon} D(B)
$$

with

$$
\hat{\epsilon} \theta=q \theta \hat{\epsilon}, \quad \hat{\epsilon} \partial_{\theta}=q^{-1} \partial_{\theta} \hat{\epsilon} \quad \hat{\epsilon} \delta_{\theta}=q^{-1} \delta_{\theta} \hat{\epsilon} .
$$

Unlike $\epsilon$, this new parameter $\hat{\epsilon}$ commutes with $Z$ and $\dot{Z}$. Therefore, in the particular case where $A$ is a function of $Z$ and $\dot{Z}$ only, i.e. $A \neq A(D Z)$, we have simply

$$
D(A B)=D(A) B+A D(B), \quad A=A(Z, \dot{Z})
$$


As we will see below, we must restrict ourself to Lagrangians of the form $L=$ $L(Z, D Z, \dot{Z})$, i.e., with no dependence on terms as $D^{i} Z$ with $1<i<F$. Then, a general variation of the action is written as

$$
\delta S=\int d t d \theta\left(\delta Z \frac{\partial L}{\partial Z}+\delta D Z \frac{\partial L}{\partial D Z}+\delta \dot{Z} \frac{\partial L}{\partial \dot{Z}}\right) .
$$

In order to write $\delta S$ such as in (63), all the variables must commute under the $\theta$-integration, i.e., we need (50) and (51). However, in general there is no term

such as $\dot{Z} Z$, either in free part (49) or in the interaction part (see below), so we can forget (51). As usual, the last term is rewritten as $-\delta Z \partial_{t}(\partial L / \partial \dot{Z})$. Using (62) and $\int d t d \theta D(\Lambda)=0$, we can rewrite the second term as $-\delta Z D(\partial L / \partial D Z)$. Then, requiring $\delta S=0$ for arbitrary variations $\delta Z$ leads to the following Euler-Lagrange equation:

$$
\frac{\partial L}{\partial Z}-D\left(\frac{\partial L}{\partial D Z}\right)-\frac{d}{d t}\left(\frac{\partial L}{\partial \dot{Z}}\right)=0 .
$$

Note that, as mentioned before, $\dot{Z}$ and $D Z$ must be considered as independent variables even for $F=1$. We now see why we must consider only Lagragians of the form $L=L(Z, D Z, \dot{Z})$, since the least-action principle yields the equation of motion only through the Leibniz rule. The formula (64) is a generalization of the superspace Euler-Lagrange equation of Ref. [6].

For the present free-particle case, the equation of motion is trivial. Indeed, from (43) and (64), we obtain $\dot{\Pi}=0$, i.e., $\ddot{x}=0$ and $\dot{\psi}_{(i)}=0$ for $i>0$. However, recalling that $Z, D Z$ and $\dot{Z}$ are fractional superfields and that any product of such fields is again a fractional superfield, it is straightforward to add magnetic interactions which preserve the FSUSY invariance. ${ }^{[4]}$ For instance (in more than one dimension), a term such as $A_{i}(Z) D Z_{i}$ leads, after integration over $\theta$, to $L_{\text {int }}=$ $A_{i}(x) \dot{x}_{i}+$ spin-magnetic-field couplings.

\section{Concluding remarks}

As mentioned in the introduction, the relation $Q^{F}=-i \partial_{t}$ is inspired from the FSUSY quantum mechanics $\hat{Q}^{F}=\hat{H}$, where $\hat{Q}$ is the quantum fractional supercharge and $\hat{H}$ the Hamiltonian. ${ }^{[1,2]}$ However, there is another generalization of SUSY quantum mechanics called para-supersymmetric (PSUSY) quantum mechanics ${ }^{[7]}$. For instance, PSUSY quantum mechanics of order 3 is defined 
through the relation $\hat{Q}^{3}=\hat{Q} \hat{H}$ with $\hat{Q}^{2} \neq \hat{H}$. Correspondingly, there is a generator of PSUSY transformations, $Q=\partial_{\theta}-i e \theta \partial_{t}$ with $\theta$ a paragrassmann variable of order 3 (and $e^{-1}=3 \alpha$ ), which satisfies $Q^{3}=-i Q \partial t$ (but $Q^{2} \neq-i \partial t$ ). ${ }^{[5]}$ The para-superspace coordinate is of the form $Z(t, \theta)=x+\theta \psi+\theta^{2} B$, where $B$ is a bosonic auxiliary field since the theory has only two sectors. ${ }^{[4]}$

\section{Acknowledgments}

I am pleased to thank Keith Dienes for very valuable discussions and comments. This work is supported in part by a fellowship from the Natural Sciences and Engineering Research Council (NSERC) of Canada.

\section{References}

[1] S. Durand, "Fractional Supersymmetry and Quantum Mechanics", preprint McGill/92-54 (April 1993) hep-th/9305128, to appear in Phys. Lett. B312, 115 (1993).

[2] S. Durand, "Extended Fractional Supersymmetric Quantum Mechanics", preprint McGill/93-03 (April 1993) hep-th/9305129, to appear in Mod. Phys. Lett. A8, 1795 (1993).

[3] S. Durand, "Fractional Supersymmetry", preprint McGill/93-05 (March 1993) to appear in the Proceedings of the VII J.A. Swieca Summer School on Particles and Fields, Campos do Jordão, Brazil, January 1993 (World Scientific, 1993).

[4] S. Durand, in preparation.

[5] S. Durand, Mod. Phys. Lett. A7, 2905 (1992).

[6] E. D'Hoker and L. Vinet, Lett. Math. Phys. 8, 439 (1984).

[7] V.A. Rubakov and V.P. Spiridonov, Mod. Phys. Lett. A3, 1337 (1988); S. Durand, M. Mayrand, V.P. Spiridonov and L. Vinet, Mod. Phys. Lett. A6, 3163 (1991). 